\title{
Videoüberwachung im öffentlichen Raum
}

\section{Einleitung}

Technische Entwicklungen lösen Aufgaben; den Juristen schaffen sie sie. Der Einsatz von Videokameras zur Überwachung öffentlichen Raumes macht dabei keine Ausnahme. Nachdem die Technik ausgereift, die Anschaffungspreise gesunken und die Fakten geschaffen sind, ziehen Gesetzgeber, Literatur und Rechtsprechung in den üblichen Intervallen nach.

Bei der rechtlichen Beurteilung der Videoüberwachung hat sich schnell ein Konsens gebildet: Formell sei die Gesetzgebungskompetenz der Länder zweifelhaft; materiell sei das Recht auf Informationelle Selbstbestimmung Maßstab, ${ }^{\mathrm{I}}$ wobei gelegentlich Zweifel an der Geeignetheit geäußert werden. Übersichtsaufnahmen hätten keine Eingriffsqualität, weil keine personenbezogenen Daten erhoben würden. ${ }^{2}$

Doch schon einfache Kontrollüberlegungen wecken Zweifel: Versammlungen dürfen wegen der ihnen unterstellten Sensibilität nach \ I 2a VersG nur unter engen Voraussetzungen gefilmt werden. Müssen dauerhaft installierte Kameras in Fußgängerzonen abgeschaltet werden, wenn dort Versammlungen - etwa auch spontan - stattfinden ? ${ }^{3}$ Was rechtfertigt die Annahme, dass allein die Versammlungsfreiheit besonders sensibel sei, obwohl gerade sie öffentlich ausgeübt wird? Defekte Kameras übertragen überhaupt keine Informationen; ist ihre sichtbare Existenz, scheinbare Funktion und die davon ausgehende Wirkung grundrechtlich völlig unbeachtlich?

Dass die allenthalben präsentierte Prüfung Videoüberwachung zu tolerieren geneigt ist, überrascht kaum. Sie erfasst lediglich Teilaspekte des grundrechtlichen Schutzprogramms (A.). Soweit erkennbar bislang ausgeblendet ist die richterliche Kontrolle der Videoüberwachung. Ursache ist die fragwürdige Prämisse, Videoüberwachung finde »offen« statt. Diese Annahme ist genauer zu untersuchen und mit ihr die Erfordernisse effektiven Rechtsschutzes (B.). Doch auch in anderer Hinsicht scheint die Videoüberwachung über sicher geglaubte dogmatische Einsichten erhaben: Während polizeiliche Maßnahmen bislang durch einen Verwaltungs- oder Realakt und einen Adressaten spezifiziert und als solche Prüfungsgegenstand waren, verursacht die Dauerüberwachung Unschärfen, die zu verkürzter Prüfung verleiten (C.). Eine genaue Feststellung des Prüfungsgegenstandes weckt auch weitere Zweifel an der Verfassungsmäßigkeit der Ermächtigungsgrundlagen. Deren Tatbestand nämlich ist unvollständig und wird durch Verwaltungsvorschriften ergänzt - ein wohl ungenügendes Regelungsniveau für Grundrechtseingriffe (D.).

\section{A. Grundrechtliche Maßstäbe der Videoüberwachung}

Materieller Prüfungsmaßstab der Videoüberwachung öffentlichen Raumes ist nach verbreiteter Vorstellung allein das Recht auf Informationelle Selbstbestimmung. Es steht außer Zweifel, daß dieses Recht staatlicher Beobachtung und Datenerhebung

I VG Karlsruhe, NVwZ 2002, S. I I7; Scholand, DuD 2000, S. 202, 203; Weichert, DuD 2000, S. 662, 662 f.; Hasse, ThürVBl. 2000, S. I69; Roggan, NVwZ 200I, S. I 34, I 35 f. und Waechter, NdsVBl. 200I, S. 77.

2 VG Karlsruhe, NVwZ 2002, S. I I7, I I 8; VG Halle, LKV 2000, S. I64, I65; Hasse, ThürVBl. 2000, S. I69.

3 So die Dienstanweisung des Polizeipräsidiums Mannheim, vgl. VG Karlsruhe, NVwZ 2002, S. I I7, I I 9 . Der Hessische Gesetzgeber hat für diesen Fall mit $\$ I4 Abs. 2 S. I HSOG eine kompetenzwidrige - Art. 8 Abs. 2 GG - und damit nichtige Regelung schaffen. 
Grenzen setzt. Doch das BVerfG misst deren Folgen auch an anderen Grundrechten und beurteilt die Eingriffsintensität sehr differenziert.

Die Videoüberwachung stellt aber auch die Dogmatik zum Recht auf Informationelle Selbstbestimmung vor neue Aufgaben: Informationelle Selbstbestimmung schützt gegen die Erhebung und Verwendung personenbezogener Daten; werden - etwa wegen eines Defektes der Kamera - keine Daten erhoben oder wegen der Bildvergrößerung nur Daten erfasst, die keine Identifizierung ermöglichen, greift das Recht auf Informationelle Selbstbestimmung nach verbreiteter Überzeugung nicht. ${ }^{4}$ Die Betroffenen können aber nicht beurteilen, ob personenbezogene Daten erhoben werden, und unterliegen daher stets den Wirkungen der »Beobachtung «. Für die demonstrative Aufstellung von Kameraattrappen bei einer Versammlung würden hieran wohl kaum Zweifel geäußert. Diese Überlegung zeigt, dass es nicht allein auf die tatsächliche Erhebung von Informationen ankommt.

Dadurch entstehen zwei miteinander verbundene Fragen: Welche Grundrechte sind in welcher Intensität betroffen? Und: Welche Bedeutung haben vermeintliche Eingriffe? Dazu haben Rechtsprechung und Literatur unterschiedliche Lösungsansätze entwickelt.

\section{Verbaltensänderungen durch Informationszugriff in der Rechtsprechung des $B \operatorname{VerfG}$}

Dass Beobachtung Rückwirkung auf das Verhalten und damit die Grundrechtsausübung hat, ist empirisch und psychologisch's erforscht und auch in Rechtsprechung und Literatur seit langem anerkannt. ${ }^{6}$ Das BVerfG hat verhaltensändernde Effekte zunächst unter dem Schutzbereich der Privatsphäre erfasst und sie an Art. 2 Abs. I i.V.m. I Abs. I GG angebunden. Als sich die Beschreibung dieses Schutzbereiches später als unzureichend erwies, schuf es in ähnlichem Ansatz das Recht auf Informationelle Selbstbestimmung.

Doch das BVerfG hat auch andere Grundrechte herangezogen: Schutz gegen Zugriff auf telefonisch übertragene Informationen sieht es durch Art. Io Abs. I GG gewährleistet, gegen Informationszugriffe auf Redaktionen hat es Art. 5 Abs. I S. 2 GG ins Feld geführt und für die Beobachtung von Demonstrationen auf Art. 8 Abs. I GG verwiesen.

\section{Recht auf Informationelle Selbstbestimmung und Privatsphäre}

In der Mikrozensus-Entscheidung hat das BVerfG betont, dass bereits die bewertungsneutrale Einsichtnahme des Staates psychischen Druck auslösen könne, der die freie Entfaltung der Persönlichkeit zu hemmen vermag.7 Explizit ausgesprochen hat das Gericht dies zunächst für den »Bereich privater Lebensgestaltung «, einen Kernbereich der Grundrechte, der auf eine Gesamtschau von Art. I Abs. I i.V. m. Art. 2 Abs. I GG gestützt wurde.

Doch auch innerhalb der Privatsphäre besteht Schutz gegen unterschwellige Verhaltensänderungen durch Informationszugriffe. ${ }^{8}$ Dieser Schutz der Privatsphäre ist nicht

\footnotetext{
4 VG Karlsruhe, NVwZ 2002, S. I I7, I I 8; VG Halle, LKV 2000, S. I64, I65; Hasse, ThürVBl. 2000, S. I69.

5 Zur psychologischen Erklärung solcher Verhaltensanpassungen vgl. Nachweise bei Geiger, Verfassungsfragen zur polizeilichen Anwendung der Videoüberwachung bei der Straftatenbekämpfung, I993, S. 55 ff.

6 BVerfGE 27, I, 6 f.; 65, I, 42 f. und 100, 31 3, 359/38 I. In der Literatur zuerst Evers, Privatsphäre (1960) S. 38 ff.; auch Geiger (Fn. 5), S. 55; Dolderer, NVwZ 200I, S. I30, I32.

7 BVerfGE 27, I, $6 \mathrm{f}$.

8 BVerfGE 27, 344; 32, 373; 34, 238; 44, 353; IOI, 36I, 382.
} 
nur Zweck, sondern zugleich Mittel zu anderen Zwecken. Der Verzicht auf Infor-

mationszugriffe ist - so das BVerfG - Voraussetzung für die Verwirklichung anderer grundrechtlich geschützter Interessen. ${ }^{9}$ Der Wert dieser Interessen bestimmt das Ergebnis der Abwägung. ${ }^{10}$

Das Volkszählungsurteil ${ }^{11}$ hat diese Überlegungen abermals erweitert: Ausdrücklich wird die Privatheit als tatbestandliches Erfordernis ganz aufgegeben. Auch offen einsehbares Verhalten nämlich - als Beispiele nennt das Gericht die Teilnahme an einer Versammlung oder Bürgerinitiative - sei gegenüber staatlicher Beobachtung empfindlich. Wegen der neuen technischen Möglichkeiten der Informationsverarbeitung könnten Verhaltensunsicherheiten entstehen, die den Einzelnen dazu bewegten, auf die Ausübung seiner Grundrechte zu verzichten. ${ }^{12}$ Dieser Folge begegne das Recht auf Informationelle Selbstbestimmung.

Konstruktiv weicht dies nur geringfügig von der Privatsphärenkonzeption ab: Verhaltensdeformierende Wirkungen werden - gleich welcher grundrechtliche Sachbereich betroffen ist - nicht als besondere Form der Beeinträchtigung dieses Grundrechts, sondern als Eingriff in ein anderes Grundrecht behandelt. Die Sensibilität und Verwendungsmöglichkeit der Information beeinflusst die Interessenabwägung im Rahmen der Verhältnismäßigkeitsprüfung. ${ }^{13}$

\section{Lösungen unter Art. I० Abs. I GG}

Im jüngsten Abhörurteil ${ }^{14}$ hat sich das BVerfG abermals mit verhaltensverändernden Wirkungen staatlicher Informationszugriffe befasst. Hier hat es Schutz gegen diese Folgen in Art. ıo Abs. I GG verankert gesehen: Er solle verhindern, »dass der Meinungs- und Informationsaustausch mittels Fernmeldeanlagen deswegen unterbleibt oder nach Form und Inhalt verändert verläuft«, weil die Beteiligten damit rechnen müssen, dass staatliche Stellen Kenntnisse über Kommunikationsbeziehungen oder -inhalte gewinnen. ${ }^{\text {is }}$

Auch hier betont das BVerfG, dass die Verwirklichung bestimmter Grundrechte und damit verbundener Gemeinschaftsinteressen den Verzicht auf Informationszugriffe voraussetzt. Art. Io GG sei gegenüber dem Recht auf Informationelle Selbstbestimmung spezieller, wenn Informationen telefonisch übertragen werden. ${ }^{16}$

Der Informationszugriff kann neben der Fernmeldefreiheit auch andere Grundrechte beeinträchtigen. ${ }^{17}$ Der Schutz gegen Informationszugriffe durch Art. I० Abs. I GG ist daher nicht abschließend; wegen des Spezialitätsverhältnisses gilt dies entsprechend für die informationelle Selbstbestimmung.

Schließlich hat das BVerfG einen Eingriff in Art. Io Abs. I GG nicht erst in der tatsächlichen Informationserhebung gesehen. Wenn der Grundrechtseingriff nicht bemerkbar sei, werde die Eingriffsschwelle bereits durch die Befürchtung der Überwachung überschritten. ${ }^{18}$

9 BVerfGE Iо1, 361, 382.

Io BVerfGE 44, 353, 353 (LS I) und $378 \mathrm{ff}$.

I I BVerfGE 65, I.

I 2 BVerfGE 65, I, 43.

I 3 BVerfGE 65, I, $44 \mathrm{f}$.

I4 BVerfGE IO0, 3 I 3 .

Is BVerfGE I00, 3 I 3, 359 und 38 I. Ähnlich bereits für das nichtöffentlich gesprochene Wort BVerfGE 34, 238, 246 f. auf der Grundlage von Art. 2 Abs. I, Art. I Abs. I GG.

I6 BVerfGE I00, 31 3, 358.

I7 BVerfGE Iо0, 31 3, 365; in dieser Entscheidung war es die Pressefreiheit, vgl. dazu sogleich 3.

I 8 BVerfGE I00, 31 3, 38I. 
Informationelle Selbstbestimmung und Art. I० Abs. I GG sind Instrumente, Verhaltensfreiheit generell zu sichern. Daneben hat das BVerfG andere Grundrechtsgarantien gegen den Verhaltenswandel in ihren speziellen Sachbereichen herangezogen. Entwickelt wurde dies in der Rechtsprechung zum Informationszugriff auf Presseund Rundfunkredaktionen: Die Befürchtung, dass der Staat die vorausgesetzte Vertraulichkeit brechen könnte, führe dazu, dass Informanten ihr Wissen nicht an die Presse weitergäben; dadurch, so das BVerfG, würde das Grundrecht der Pressefreiheit der Redaktionen verletzt. ${ }^{19}$

In diesem Ansatz siedelt das BVerfG den Schutz gegen Verhaltensänderungen direkt bei dem Grundrecht an, dessen Verwirklichung unter dem Informationszugriff leidet. Auch hier berücksichtigt es nicht nur das tatsächliche Auskunftsverlangen, sondern schon die berechtigte Erwartung des Zugriffs.

In ähnlicher Tendenz hat das BVerfG exzessive Beobachtung und Registrierung von Versammlungsteilnehmern für unvereinbar mit Art. 8 Abs. I GG erklärt. ${ }^{\circ}$ Dabei misst es die Befürchtung von Nachteilen ebenfalls an dem Grundrecht, dessen Ausübung von der Verhaltensänderung betroffen ist.

\section{Exkurs: Rechtsprechung des BVerfG zur Einschüchterung bei der Meinungsfreibeit}

Das BVerfG hat Verhaltensänderungen durch Einschüchterung noch in einem weiteren Zusammenhang, nämlich der Rechtsprechung zur Meinungsfreiheit, thematisiert. Fachgerichte hatten wegen kritischer bis herabsetzender Meinungsäußerungen Schadenersatzansprüche bzw. Kriminalstrafen ausgesprochen. ${ }^{21}$ Das BVerfG hat daraufhin - zuerst in einem Sondervotum der Richterin Rupp-v. Brünneck, ${ }^{22}$ deren Thesen wenig später übernommen wurden ${ }^{23}$ - die einschüchternde Wirkung solcher Sanktionen auf die Meinungsäußerung anerkannt. Die Befürchtung, dass an eine Meinungsäußerung ein Nachteil geknüpft werde, entfalte eine generalpräventive Wirkung und führe zu Selbstzensur. ${ }^{24}$

Diese Fälle sind den bisher besprochenen ähnlich: Auch hier - so die Hypothese über den Kausalverlauf - sehen Personen aus Furcht vor Nachteilen von der Grundrechtsausübung ab; die Ungewissheit der Konsequenzen einer Grundrechtsausübung hat generalpräventive Wirkung. Diese generalpräventive Wirkung könne die Meinungsfreiheit »in ihrer Substanz « treffen. ${ }^{25}$ Als Konsequenz hat das BVerfG die Kontrolldichte erhöht. ${ }^{26}$

\section{Rechtsprechung anderer Gerichte}

Andere Gerichte haben ebenfalls gelegentlich die einschüchternde Wirkung staatlicher Beobachtung erörtert.

Bei einem Strafprozess waren »die Zuhörer des Verfahrens bei ihrer Ankunft, während ihres Aufenthalts vor dem Gericht, vor dem Gerichtssaal oder anderweitig

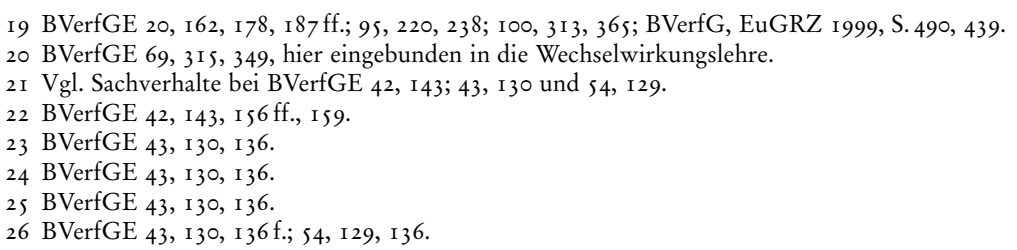


im Gerichtsgebäude « von Beamten der Kriminalpolizei fotografiert worden; auch ein

Videorecorder und Richtmikrophone sollen eingesetzt worden sein. ${ }^{27}$ Der BGH hat eingeräumt, dass die Öffentlichkeit der Hauptverhandlung beeinträchtigt sein könne, wenn »von Maßnahmen staatlicher Organe ein starker psychischer Druck dadurch ausgeht, dass diese in dem unbefangenen Interessenten den Eindruck einer realen Gefahr entstehen lassen, der Besuch der Hauptverhandlung könne für ihn konkrete Nachteile nach sich ziehen. $\ll^{28}$ Im dargestellten Fall hat der BGH eine solche Wirkung zwar nicht angenommen; die Behauptung, einige Personen hätten unter den geschilderten Umständen auf die Teilnahme an der Hauptverhandlung verzichtete, sei zu unbestimmt. Allerdings erkennt auch der BGH an, dass schon die Befürchtung der Betroffenen rechtlich erheblich ist.

Die Entscheidung weist auf ein Dilemma der einschüchternden Wirkungen hin: Die primär Betroffenen, auf die die Einschüchterung wirkt, wollen gegenüber staatlichen Stellen gerade nicht in Erscheinung treten und verzichten auf eine gerichtliche Kontrolle der Maßnahmen. Die mittelbar Betroffenen - in diesem Falle der Revisionsführer - sind nicht »selbst « betroffen und deshalb nicht klagebefugt oder sie können den Effekt nicht belegen. Wie stark dieser Effekt dennoch ist, zeigen Großkundgebungen gegen Atomanlagen oder Castor-Transporte: Die zu erwartende Beobachtung führt zu einer Selektion der Teilnehmer - wer keinen Ärger riskieren möchte, bleibt zu Hause.

Das OVG Bremen hatte über die Rechtmäßigkeit offener Videoaufnahmen eines Demonstrationszuges zu entscheiden. ${ }^{29}$ Es hat diese Aufzeichnungen nicht am Recht auf Informationelle Selbstbestimmung gemessen - in das sie nach Ansicht des OVG Bremens ebenfalls eingriffen -, sondern an Art. 8 Abs. I GG selbst: Die Versammlungsfreiheit gewährleiste auch das Recht, dies ohne staatliche Beeinflussung tun zu können. Überwachung und Registrierung beeinträchtigten Durchführung und Teilnahme an einer Demonstration, denn wer mit Risiken rechne, werde möglicherweise auf die Ausübung der Grundrechte verzichten. Art. 8 Abs. I GG schütze auch davor, »das Grundrecht im Visier von Polizei und Verfassungsschutz wahrnehmen zu müssen $\ll .3 \circ$

\section{Lösungsansätze in der Literatur}

Verhaltensdeformationen haben auch die Literatur gelegentlich beschäftigt. Einige Beiträge betreffen die Selbstzensur bei Meinungsäußerungen als Folge erwarteter Nachteile, andere behandeln das Problem der Verhaltensänderung durch Informationszugriffe allgemein. Durchweg zeigt sich eine große Sensibilität für unterschwellige Verhaltensänderungen. Der verhaltensbeeinflussende Effekte wird dabei selbst als Eingriff gewertet oder der »objektiv-rechtlichen« Seite zugewiesen und so in die Abwägung einbezogen.

\section{Verhaltensdeformation als Eingriff in spezielle Grundrechte}

Speziell für Art. 8 Abs. I GG nimmt die Literatur ganz überwiegend an, dass schon die Beobachtung in den Schutzbereich eingreife; der Versammlungsfreiheit enthalte eine Komponente »innerer Versammlungsfreiheit «. ${ }^{31}$ Das Problem wird dabei auf die

27 Sachverhalt nach BGH, NJW I980, S. 249.

28 BGH, NJW I980, S. 249.

29 OVG Bremen, NVwZ i 990 , S. i 88 f.

30 OVG Bremen, NVwZ i990, S. i 88 , i 89.

3 I Ridder/Hase, Versammlungsrecht. Kommentar, $\$$ I2a, Rn. I8; Dietl et al., Demonstrations- und Ver-

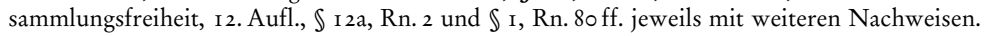


Frage verschoben, welche Beobachtungsintensität für einen Eingriff in die »innere Versammlungsfreiheit « gegeben sein muss. Analog will Dolderer jedenfalls in der »strategischen « Überwachung öffentlichen Raumes durch Videokameras einen Eingriff in Art. 2 Abs. I GG sehen; solches Zeigen von »Polizeipräsenz« soll »psychologisch vermittelte Eingriffswirkungen « entfalten. ${ }^{32}$ Garstka ist der Auffassung, Art. I I Abs. I GG gewährleiste nicht nur die Möglichkeit, sich frei zu bewegen, sondern auch, dass dies nicht festgehalten werde. ${ }^{33}$ Verhindert werden müsse deswegen die »flächendeckende Videoüberwachung öffentlicher Räume«.34

Vergleichbare Annahmen vertritt ein Teil der Literatur auch für andere Sachbereiche: Nach Schwan stellen Informationseingriffe »nicht nur Gefährdungen anderer Freiheitspositionen dar, sondern sie beschneiden selbst und unmittelbar die grundrechtlich verbürgte Freiheit«.35 Ähnlich Schlink: »Wo das staatliche Informationsgebaren die gesellschaftlichen Kommunikationen und Interaktionen in spezialgrundrechtlich geschützten Lebensbereichen steuernd, hemmend und einschüchternd betrifft, da kann und muss es an den Spezialgrundrechten gemessen werden «. ${ }^{36}$ Herzog hält Beeinträchtigungen und Hemmungen - etwa das »Abhören von Meinungsäußerungen, die nicht für die Öffentlichkeit bestimmt sind «-für Eingriffsformen, die an Art. 5 Abs. I GG zu messen seien. ${ }^{37}$ Auch die Aufsicht und Dokumentation von Versammlungen will Herzog an Art. 8 Abs. I GG als speziellem Grundrecht messen, ${ }^{38}$ daneben hält er das Allgemeine Persönlichkeitsrecht für anwendbar. Auch Frowein ${ }^{39}$ schließt aus der verfassungsgerichtlichen Rechtsprechung zur generalpräventiven Wirkung für die Meinungsfreiheit, dass Rückwirkungen am beeinträchtigten Grundrecht selbst zu messen seien.

\section{Objektiv-rechtliche Lösungen}

Ein Teil der Literatur fasst die einschüchternde Wirkung nicht als eigenständigen Eingriff auf, sondern berücksichtigt die Wirkungen im Rahmen anderer Grundrechte:

Hoffmann-Riem etwa bindet einschüchternde Wirkungen durch Informationserhebung an das »objektive Prinzip« der Versammlungsfreiheit; dieses Prinzip entfalte eine »Ausstrahlungswirkung $^{\circ} .^{\circ}$

Schmitt Glaeser analysiert die Rechtsprechung des BVerfG zur einschüchternden Wirkung bei der Meinungsfreiheit. Grundrechtsbeeinträchtigungen durch Verunsicherung ließen sich » wohl nur bei der objektiv-rechtlichen Seite der Grundrechtsgewährleistung ansiedeln $\ll^{4^{\mathrm{I}}}$ Er folgert dies aus der Begründung des BVerfG, das von einer »generellen« Wirkung spricht. Ob diese Beeinträchtigungsform als Eingriff zu werten sei, sei offen.

32 NVwZ 200I, S. I30, I3 If. Das maßgebliche Kriterium - "strategisch « - wird nicht erläutert, aus der Begründung ergibt sich aber, dass die »psychologisch vermittelten Eingriffswirkungen « auch entstehen, wenn die Überwachung nicht »strategisch « ist.

33 Garstka, DuD 2000, S. 192, I93; dagegen Waechter, NdsVBl. 2001, S. 77, 79 ff.

34 Garstka, DuD 2000, S. 192, I93, Hervorhebung im Original.

35 Amtsgeheimnis, S. $23 \mathrm{f}$.

36 Amtshilfe, S. I70 (Fn. 5).

37 Maunz/Dürig, Art. s I GG (1992), Rn. 79.

38 Maunz/Dürig, Art. 8 I GG (I987), Rn. 87.

39 AöR ros (i980), S. I69, I87.

40 Alternativkommentar-GG, Art. 8 (200I), Rn. 43.

4I AöR II3 (1988), S. 52, 67. 
Die - auch nur vermutete - Videobeobachtung greift danach mindestens in das Recht auf Informationelle Selbstbestimmung ein. Für die Interessenabwägung ist maßgeblich, welche Intensität dieser Eingriff hat. Die verbreitete Annahme, der Eingriff sei relativ harmlos ${ }^{42}$ und werde von der Mehrheit sogar begrüßt,43 beruht auf zweifelhaften und unvollständigen Annahmen: Es wird behauptet, dass Eingriffe nur »Verdächtige «träfen. Andere maßgebliche Faktoren - etwa die Zahl der Betroffenen werden dagegen nicht erörtert.

Nach der Rechtsprechung des BVerfG ist für die Intensität eines Eingriffs maßgeblich, ob er mit dem Verhalten des Betroffenen in Beziehung gebracht werden kann oder durch ihn veranlasst ist. ${ }^{44}$

Bei der Videoüberwachung - so eine verbreitete Ansicht - fänden Aufzeichnungen und damit Eingriffe nur dann statt, wenn ein »Anfangs- oder Gefahrenverdacht «4s bestehe. Dieser Annahme liegen indes völlig neue Verdachtsbegriffe zugrunde: Welche Handlungen »verdächtig « sind, hängt nämlich von der Beobachtungsmethode ab: Bei Übersichtsaufnahmen sind strafbare Handlungen als solche gar nicht erkennbar. Die »Verabredung und Vorbereitung von Straftaten« lässt sich nicht von anderen Gesprächen unterscheiden, der Handel mit Betäubungsmitteln oder ein Diebstahl zeichnen sich kaum jemals als solche auf dem Monitor ab, und einen flüchtenden Täter von jemand zu unterscheiden, der einen Bus zu erreichen versucht, dürfte - zumal ohne Tonübertragung - auch bei großer »kriminalistischer Erfahrung « unmöglich sein. »Verdächtig « wird damit auch Verhalten, das keine zureichenden tatsächlichen Anhaltspunkte im herkömmlichen Sinne bietet. Die Detailbeobachtung und Aufzeichnung trifft deshalb bereits aus tatsächlichen Gründen zu einem erheblichen Anteil Nichtstörer. Das erhöht die Eingriffsintensität jedenfalls nach ständiger Rechtsprechung des BVerfG.

Bei der Bewertung der Eingriffsintensität berücksichtigt das BVerfG nicht allein die Folgen für den individuellen Beschwerdeführer, sondern auch, wie viele Grundrechtsträger die Maßnahme außerdem erfaßt. ${ }^{6}{ }^{6}$ Dabei hat es gerade für unbemerkbare Maßnahmen angenommen, daß diese Zahl sehr hoch sei. Es müsse auch die Wirkung auf die Gesellschaft insgesamt betrachtet werden, zumal wenn das beeinträchtigte Verhalten einen besonderen Gemeinwohlbezug aufweise. ${ }^{47}$

\section{Zwischenbilanz}

Videoüberwachung ist am Recht auf Informationelle Selbstbestimmung zu messen; die durch den Zugriff beeinträchtigten Interessen sind im Rahmen der Güterabwägung zu berücksichtigen. Daneben sind selbständig spezielle Grundrechtsgarantien heranzuziehen, wenn ein Verhaltenswandel die Verwirklichung der durch sie geschützten Werte beeinträchtigt.

Ein Eingriff in diese Grundrechte setzt keine tatsächliche Informationserhebung voraus; weil diese nicht bemerkbar ist, genügt es, dass der Grundrechtsträger mit einem Zugriff und damit verbundenen Nachteilen rechnen muss.

42 VG Karlsruhe, NVwZ 2002, S. I I7, i 18. Das VG Halle, LKV 2000, S. I64, I65 meint sogar, dass sie keinen Eingriff darstelle, weil überwiegend keine Aufzeichnung stattfinde.

43 VG Karlsruhe, NVwZ 2002, S. i i7, i 8.

44 BVerfGE Iо0, 3 1 3, 380.

45 VG Halle, LKV 2000, S. I64, I64.

46 BVerfGE I00, 3I 3, $38 \mathrm{I}$.

47 BVerfGE I00, 31 3,38 I. 
Die Eingriffstiefe von Videoaufzeichnungen ist erheblich: Maßgebend ist dabei, dass sie in großem Umfang Nichtverantwortliche und Nichtverdächtige trifft, dass viele Grundrechtsträger in verschiedenen Handlungsweisen betroffen sind und Verhalten mit Gemeinwohlbezug gehemmt wird.

\section{B. Videoüberwachung und Rechtsschutzgarantie}

\section{Offenheit der Videoüberwachung?}

Videoüberwachung erfolge offen, weil die Kameras sichtbar und Hinweisschilder aufgestellt seien - so eine verbreitete Annahme..$^{8} \mathrm{Ob}$ sichtbare Aufstellung schon zu offener Beobachtung führt, ist aber zweifelhaft: Polizeiliche Handlungen sind durch Maßnahmetyp und Adressaten bestimmt. Offenheit bedeutet, dass der Adressat weiß, dass er zu einem bestimmten Zeitpunkt Ziel einer polizeilichen Maßnahme ist - wie bei Platzverweis, Befragung oder Identitätsfeststellung. Hinweisschilder auf eine Videoüberwachung in einem bestimmten Bereich weisen nur darauf hin, dass dort generell Maßnahmen bestimmter Art durchgeführt werden. Wer zu einem konkreten Zeitpunkt als vermeintlicher Störer erfasst wird, erfährt dies nicht - also ist die Maßnahme, die den Grundrechtseingriff auslöst, verdeckt.49 Genügte die generelle Erklärung, dass bestimmte Maßnahmen durchgeführt werden, würde bereits die gesetzliche Ermächtigung die Verdecktheit von Eingriffen ausschließen.

\section{Rechtfertigung beschränkten Rechtsschutzes}

Wird jemand durch die öffentliche Gewalt in seinen Rechten verletzt, so steht ihm der Rechtsweg offen. Diese Garantie des Art. I9 Abs. 4 S. I GG meint effektiven Rechtsschutz. In tatsächlicher Hinsicht setzt dies Kenntnis von einer belastenden Maßnahme voraus. ${ }^{\circ}$ Die Kenntnis von einer Filmaufnahme fehlt dem Betroffenen aber selbst dann, wenn er von der Videoüberwachung eines Straßenzuges weiß, so dass der Rechtsschutz eingeschränkt ist. ${ }^{\text {s }}$

Eine solche Einschränkung ist im Interesse konkurrierender Verfassungsgüter möglich.52 Das setzt aber voraus, dass die Verheimlichung erforderlich ist. Wo verdeckte Maßnahmen für zulässig gehalten werden, sind sie wegen der spezifischen Umstände meist das einzig erfolgversprechende Mittel zur Informationsbeschaffung. ${ }^{53}$ Nicht so die Videoüberwachung: Sie wird gerade als Äquivalent einer direkten Beobachtung dargestellt. ${ }^{54}$

Eine Rechtfertigung setzt daneben die Angemessenheit des Mittels im Verhältnis zum Zweck voraus. 5 s Nicht jedes beliebige Interesse rechtfertigt einen Eingriff in Art. I 9 Abs. 4 GG: Mag eine verdeckte Informationserhebung zum Staatsschutz, zur Abwehr von Gefahren für hochrangige Rechtsgüter oder zur Verfolgung schwerer Straftaten zulässig sein - die Dokumentation vermuteter Straftaten jeder Art oder »nicht

48 VG Karlsruhe, NVwZ 2002, S. I I7, i 8 und Hasse, ThürVBl. 2000, S. I69, I73. Ebenso die irreführende amtliche Überschrift des $\ 2$ I BawüPolG; der Tatbestand der Ermächtigungsgrundlage enthält keine Vorschriften darüber, welche Voraussetzungen für eine Offenheit gegeben sein müssen. Art. 32 Abs. 2 S. I BayPAG enthält den Begriff »offen « als Tatbestandsmerkmal.

49 So auch BVerwG, DVBl. I989, S. 200, 20 I für eine allgemein angekündigte, aber nicht konkret erkennbare Videoüberwachung.

50 BVerfGE 100, 31 3, 364; SächsVerfGH DVBl. I996, S. I423, I435; Guttenberg, NJW I993, S. 567, 574.

5 I Aus dieser Einsicht erklärt sich die Regelung des $₫ 24$ a Abs. 4 BlnASOG.

52 BVerfGE IOI, I06, I24.

53 Guttenberg, NJW i993, S. $567,575$.

54 Etwa VG Halle, LKV 2000, S. I64, I65.

55 BVerfGE I0I, 106, I 25 . 
geringfügiger Ordnungswidrigkeiten « ${ }^{6}$ rechtfertigt eine solche Einschränkung der

Rechtsschutzgarantie nicht.

\section{Mitteilungspflichten}

Polizeiliche Maßnahmen dürfen zunächst verborgen bleiben, um ihren Erfolg nicht zu gefährden; das bedeutet nicht, dass sie auch nach Erledigung des Zwecks geheim gehalten werden dürfen. Bei unerkennbaren Eingriffen hat das BVerfG schon eine eingeschränkte Mitteilungspflicht für unverhältnismäßig gehalten.57 Die Regelungen der Landespolizeigesetze zur Videoüberwachung sehen - mit Ausnahme der Berliner Vorschrift ${ }^{58}$ - überhaupt keine Mitteilungspflichten vor, auch dann nicht, wenn die beobachtete Person identifiziert worden ist. Solche Regelungen haben vor Art. I9 Abs. 4 GG keinen Bestand. 59

\section{Protokollierungspflichten}

Effektiver Rechtsschutz setzt weiter voraus, dass dem Gericht die zur Beurteilung notwendigen Informationen vorliegen. ${ }^{60}$ Art. I9 Abs. 4 GG verbietet daher Maßnahmen, die den Rechtsschutz vereiteln, namentlich die Löschung der erhobenen Informationen. ${ }^{61}$ Eine verfassungsgemäße Regelung enthält etwa $\ 7$ Abs. 4 S. 4 f. G-ı。: Danach werden nicht benötigte Daten gesperrt, aber für eine eventuelle gerichtliche Prüfung vorübergehend aufbewahrt. Die Befugnisse zur Videoüberwachung sehen die Löschung dagegen nach gewissem Zeitablauf auch dann vor, wenn die Informationen verwertet worden sind - nach der Rechtsprechung des BVerfG ist gerade dies verfassungswidrig. ${ }^{62}$

\section{Prüfungsgegenstand der»Videoüberwachung»}

Die Fachgerichte haben Klagen gegen Videoüberwachung bisher abgewiesen. Dies ist nicht zuletzt Ergebnis eines unvollständigen Prüfungsprogramms:

Polizeimaßnahmen sind durch einen Adressaten, einen Verwaltungs- bzw. Realakt und Zeit und Ort der Anwendung bestimmt. »Videoüberwachung öffentlichen Raumes « meint: »Überwachung von Menschen im öffentlichen Raum«.

Das VG Karlsruhe hat seiner Entscheidung einen anderen Prüfungsgegenstand zugrundegelegt: Es prüft dem Worte und der Sache nach die Rechtmäßigkeit einer Anlage, ${ }^{63}$ die sich bestenfalls als eine Prüfung der Rechtmäßigkeit ihres Dauerbetriebs interpretieren lässt. Es gibt keinen Adressaten und keinen zeitlichen Rahmen - die Konturen der polizeilichen Maßnahme verschwimmen. Das Gericht prüft sie für Zeiträume, in denen der Kläger gar nicht betroffen war, und entscheidet über die Rechtmäßigkeit der »Maßnahme« in der Zukunft. Auf die Zukunft erstreckt sich aber weder die Klage - vorbeugender Rechtsschutz war nicht beantragt -, so dass darüber auch kein Urteil ergehen durfte, $\mathbb{S} 88 \mathrm{VwGO}$. Das Gericht könnte die zukünftige Zulässigkeit einer polizeilichen Maßnahme auch gar nicht aussprechen. Für welchen

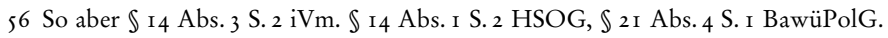

57 BVerfGE I00, 313, 398.

$58 \int 24$ a Abs. 4 BlnASOG.

59 BVerfGE 100, 31 3, 398; SächsVerfGH, DVBl. 1996, S. I423, I435.

60 BVerfGE 65 , I, 70; 69, I, 49.

6I BVerfGE I00, 3 I $3,400$.

62 BVerfG I00, 31 3, $398 \mathrm{ff}$.

63 Das VG Karlsruhe spricht von der »Rechtmäßigkeit der streitigen Anlagen« und einem »Anspruch auf Untersagung bzw. Beseitigung der Videoanlagen«, NVwZ 2002, S. I I7, I I7, I I9. 
Zeitraum gilt sein Urteil, wann endet die materielle Rechtskraft, die eine erneute Klage nach $\int$ I 2 I VwGO hindert? Welchen Einfluss haben veränderte tatsächliche Umstände, etwa eine Demonstration im Blickfeld der Kamera? Für diesen Zeitraum beurteilt sich die Rechtmäßigkeit einer Videoaufzeichnung nach \ I 2a VersG. ${ }^{64}$ Man stelle sich zum Vergleich eine Klage vor, die sich generell gegen die Durchführung von Identitätsfeststellungen an einem bestimmten Ort richtet. Hätte das Gericht diese Klage ebenso entschieden?

Bereits die prozessualen Probleme zeigen, dass hier ein unzutreffender Streitgegenstand geprüft worden ist. Die materiellen Fehler wiegen indes viel schwerer: Weil der Adressat der Maßnahme und seine Grundrechte durch die Festlegung des Streitgegenstandes gar nicht mehr im Blick waren, konnte das Gericht die Verhältnismäßigkeit nicht prüfen, sondern nur postulieren. Wenn sich herausstellte, dass das Bedienpersonal entgegen Zweck und Dienstanweisung wahllos Großaufnahmen der Passantinnen auf die Monitoren brächte - die Bedienung also evident rechtswidrig wäre -, könnte sich dieser Befund im Urteil des VG Karlsruhe gar nicht niederschlagen, weil es die Bedienung der Kameras und damit die maßgebliche polizeiliche Maßnahme an keiner Stelle geprüft hat. Nicht einmal die Rechtmäßigkeit der Dienstanweisung selbst hat das Gericht untersucht.

Es liegt danach wohl auf der Hand, dass das Urteil des VG Karlsruhe jedenfalls methodisch nicht zu halten ist. Wie aber ist die Rechtmäßigkeit der »Videoüberwachung « richtigerweise $\mathrm{zu}$ prüfen?

Zunächst muss diese Dauermaßnahme gedanklich zerlegt werden: Streitgegenstand kann nur die Beobachtung eines Adressaten in einem bestimmten Zeitraum sein. Dies ist prozessual erforderlich, um die Zulässigkeit der Klage und den Umfang der materiellen Rechtskraft zu bestimmen, und materiell ist es notwendig, um tatsächliche Umstände beurteilen zu können.

Ist Klagegegenstand Beobachtung und Aufzeichnung des Verhaltens eines Adressaten zu einem Zeitpunkt, kann die Ermessensausübung und dabei die Verhältnismäßigkeit der Maßnahme überprüft werden. Ein öffentliches Interesse an der Beobachtung einer konkreten Person in einer konkreten Situation könnte dann kaum mit Kriminalitätsstatistiken der »Innenstadt « über Jahreszeiträume begründet werden, ${ }^{65}$ sondern müsste sich auf ein »verdächtiges Verhalten« beziehen. Videoüberwachung einer Person in einer Fußgängerzone ließe sich ohne entsprechende Anhaltspunkte nicht allgemein mit »Lebensschutz $\ll^{66}$ rechtfertigen oder damit, daß »verrufene Orte für Normalbürger wieder zugänglich ${ }^{67}$ gemacht werden. Die zutreffende Wahl des Prüfungsgegenstandes führt so zu einer rationalen Tatsachengrundlage, die Voraussetzung für eine haltbare rechtliche Prüfung und Güterabwägung ist. Sie ermöglicht außerdem, neben der Informationellen Selbstbestimmung speziell betroffene Grundrechte zu berücksichtigen, etwa Art. 8 Abs. I GG oder Art. 5 Abs. I S. 2 GG. Da Prüfungsgegenstand die konkrete Aufzeichnung eines Adressaten ist, erklärt sich auch - wie bei anderen verdeckten Maßnahmen - das Bedürfnis nach der Bekanntgabe des Eingriffs und der Dokumentation der für die gerichtliche Prüfung erforderlichen Informationen.

64 Die Öffnung des $\$ I 2a VersG bezieht sich nur auf Vorschriften der StPO und des OWiG.

65 So aber VG Karlsruhe, NVwZ 2002, S. i i7, II 9.

66 So aber Hasse, ThürVBl. 2000, S. I97, 2 I.

67 Schmitt Glaeser, BayVBl. 2002, S. $584,588$. 
Die Feststellung des Prüfungsgegenstandes rückt die Ermächtigungsgrundlage der Videoüberwachung auch in materieller Hinsicht noch einmal ins Blickfeld. Die bisher erlassenen Befugnisnormen regeln die tatbestandlichen Voraussetzungen der Videoüberwachung in Anbindung an bestimmte Orte. Dass auch an diesen Orten jedenfalls die Bildaufzeichnung nicht unter allen Bedingungen und gegenüber jedermann zulässig ist, liegt auf der Hand. Die Gesetzestexte enthalten jedoch keine entsprechenden Regelungen über Adressat und Anlass. ${ }^{68}$ Auch die Regelungen über die Polizeipflichtigkeit greifen nicht, weil die Betroffenen meist nicht einmal Anscheinsstörer im herkömmlichen Sinne sind. ${ }^{69}$ Nach dem »Leipziger Modell «70 und in Mannheim $^{71}$ werden anlassbezogene Voraussetzungen ergänzend auf Ebene von Dienstvorschriften geregelt, in Halle nach einer »Handlungsanweisung «.72

Grundrechtseingriffe auch in die Informationelle Selbstbestimmung unterliegen dem Gesetzesvorbehalt. ${ }^{73}$ Dabei hat der Gesetzgeber »in grundlegenden normativen Bereichen, zumal im Bereich der Grundrechtsausübung, soweit diese staatlicher Regelung zugänglich ist, alle wesentlichen Entscheidungen selbst zu treffen. «74 Die Voraussetzungen von Bildaufzeichnungen von Personen hat daher der Gesetzgeber zu regeln. Dies ist ihm auch möglich, wie die Existenz abstrakt-genereller Dienst- und Handlungsanweisungen zeigt. Wegen Verstoßes gegen den Gesetzesvorbehalt dürften daher die Regelungen in $\mathbb{} 38$ Abs. 2 sächsPolG, $\mathbb{S} 32$ Abs. 2 S. I bayPAG, $\mathbb{} 24$ Abs. I blnASOG und $\ 2$ I Abs. 3 bawüPolG verfassungswidrig sein.

\section{E. Zusammenfassung}

Videoüberwachung öffentlichen Raumes greift in das Recht auf Informationelle Selbstbestimmung ein, daneben können in der konkreten Situation andere Grundrechte betroffen sein. Ein Eingriff in diese Grundrechte setzt keine tatsächliche Informationserhebung voraus; wenn diese nicht konkret bemerkbar ist, genügt es, dass der Grundrechtsträger mit einem Zugriff und einem damit verbundenen Nachteil rechnen muss. Die Intensität des Eingriffs ist erheblich, da sie in großem Umfang Nichtverantwortliche und Nichtverdächtige trifft, weil viele Grundrechtsträger in verschiedenen Handlungsweisen betroffen sind und Verhalten mit Gemeinwohlbezug gehemmt wird.

Nach den bestehenden Regelungen - mit Ausnahme der Berliner Regelung - verletzen Beobachtung und Aufzeichnung Art. I9 Abs. 4 GG: Die Videoüberwachung ist nicht deswegen eine »offene « Maßnahme, weil die Kameras sichtbar oder angekündigt sind; den maßgeblichen konkreten Grundrechtseingriff kann ein Adressat nicht erkennen. Verdeckte Maßnahmen beschränken die Rechtsschutzgarantie aus Art. I9 Abs. 4 GG. Diese Beschränkung lässt sich nicht mit dem Interesse der Ordnungswidrigkeitenverfolgung rechtfertigen. Die Rechtsschutzgarantie gebietet ferner die Mitteilung über den Eingriff an identifizierte Personen und die Protokollierung und vorübergehende Aufbewahrung der für die gerichtliche Beurteilung maßgebenden Informationen. Diesen Maßstäben werden die gesetzlichen Regelungen nicht gerecht. Die Ermächtigungsgrundlagen der Landespolizeigesetze verletzen daneben den

68 Roggan, NVwZ 200I, S. I 34, I 37 ordnet dieses Problem dem Bestimmtheitsgrundsatz zu.

$69 \mathrm{Vgl}$. dazu oben A. IV.

70 Auszugsweiser Abdruck in Die Polizei 1997, S. 8 I.

7 I Vgl. VG Karlsruhe, NVwZ 2002, S. I I7, I 19.

72 VG Halle, LKV 2000, S. I64, I64.

73 BVerfGE 65, I, 44.

74 BVerfGE 49, 89, I 26; SächsVerfGH, DVBl. I996, S. I423, I 429. 
Gesetzesvorbehalt: Sie regeln die tatbestandlichen Voraussetzungen nicht vollständig, und ihre Ergänzung durch Verwaltungsvorschriften genügt nach der Wesentlichkeitslehre des BVerfG nicht den Anforderungen des Parlamentsvorbehaltes.

Die fachgerichtliche Prüfung der Videoüberwachung hat nicht Aufstellung und allgemeinen Betrieb einer Kamera zum Gegenstand, sondern die Beobachtung eines konkreten Adressaten zu einem bestimmten Zeitpunkt. Nur dann sind die für die Prüfung maßgebenden Faktoren, nämlich der Adressat der Maßnahme und dessen beeinträchtigte Interessen mit den geschützten Rechtsgütern abzuwägen. In diese Abwägung sind nicht allgemeine Kausalitätsvermutungen und undifferenzierte Kriminalitätsstatistiken über ganze Stadtteile einzuführen, sondern Anlass der Maßnahme, Intensität des Eingriffs und Wert des geschütztes Interesses.

\title{
Günter C. Burmeister \\ Grundrechtliche Inhalts- und Schrankenbestimmung durch private Hand?
}

\author{
- Anmerkung zur Entscheidung des Bundesverfassungsgerichts
} vom 29. Januar 2003 (I BvL 20/99 und I BvR 933/OI)

\section{Einleitung}

Auf Vorlage eines Amtsgerichts (Familiengericht) sowie im Rahmen einer Verfassungsbeschwerde hatte das Bundesverfassungsgericht darüber zu befinden, ob verfassungsrechtliche Bedenken dagegen bestehen, dass bei der Geburt eines nichtehelichen Kindes nach $\$ I626a Abs. 2 BGB ausschließlich der Mutter das Sorgerecht zusteht und der Vater nach $\$ I626a Abs. I Nr. I BGB mit ihr zusammen nur dann das Sorgerecht erlangen kann, wenn die Mutter zur Abgabe einer entsprechenden gemeinsamen - Sorgeerklärung bereit ist. Mit Urteil vom 29. Januar 2003 hat es beide Regelungen im Grundsatz für verfassungsgemäß erachtet und lediglich für jene Gruppe von Vätern, deren mit dem Kind zusammen gelebte Beziehung zur Mutter bereits vor dem Inkrafttreten des Kindschaftsreformgesetzes ihr Ende gefunden hatte, eine Übergangsregelung gefordert. ${ }^{\mathrm{I}}$ Holzschnittartig zusammengefasst: \I626a Abs. 2 BGB sei verfassungsgemäß, weil bei der Geburt des Kindes eine sichere Verantwortlichkeit bestehen müsse und die Mutter die einzig sichere Bezugsperson sei, die das Kind vorfinde. ${ }^{2}$ Bei $\$ r626a Abs. I Nr. I BGB sei die Annahme des Gesetzgebers vertretbar, eine nichteheliche Mutter werde die Verweigerung der gemeinsamen Sorgeerklärung nicht als Machtmittel gegenüber dem Vater einsetzen. $^{3}$ 